\title{
Diabetic retinopathy among self reported diabetics in southern India: a population based assessment
}

\author{
V Narendran, R K John, A Raghuram, R D Ravindran, P K Nirmalan, R D Thulasirai
}

Br J Ophthalmol 2002;86:1014-1018

See end of article for authors' affiliations

Correspondence to:

Praveen K Nirmalan

Aravind Eye Care System,

LAICO, 72, Kuruvikaran

Salai, Gandhi Nagar,

Madurai, Tamil

Nadu-625020, India;

praveen@aravind.org

Accepted for publication 2 May 2002

\begin{abstract}
Aims: To estimate the prevalence of diabetic retinopathy among self reported diabetics in a population of southern India.

Methods: A cross sectional sample of subjects aged 50 years and older was selected using a cluster sampling technique from Palakkad district of Kerala state. Eligible subjects were identified through a door to door survey. Ocular examinations including visual acuity and anterior and posterior segment examinations were performed at preselected sites within clusters. History of diabetes was elicited, and height, weight, and blood pressure were measured for all subjects.

Results: Among the 5212 examined people (92\% response rate), 68 (26.2\%) of 260 people with self reported history of diabetes had diabetic retinopathy. The age-sex adjusted prevalence of diabetes among people aged 50 years and older was $5.1 \%(95 \% \mathrm{Cl} 3.9,6.3$, deff 4.33$)$ and of diabetic retinopathy among the diabetics was $26.8 \%(95 \% \mathrm{Cl}$ : 19.2, 34.4, deff 1.99). Non-proliferative diabetic retinopathy $(94.1 \%)$ was the most common form of retinopathy seen. Two eyes were blind (presenting vision $<6 / 60$ ) as a result of retinopathy.

Conclusion: Preventive strategies have to be evolved to ensure that blindness due to diabetic retinopathy does not become a public health problem in India. Further studies are required to understand the risk factors for retinopathy and vision loss in this population.
\end{abstract}

$\mathrm{T}$ here is an increasing prevalence and incidence of diabetes with increasing age ${ }^{12}$; adult diabetics are also at risk of vision threatening retinopathies. ${ }^{34}$ The absolute number of the over 60 population in India will increase from 76 million in 2000 to 137 million by $2021 .^{5}$ It is estimated that the number of diabetics in India will increase from 19 million to 57 million between 1995 and 2025 (195\% increase). ${ }^{6}$ A quarter of the world's blind population is estimated to be in India, about 9-12 million ${ }^{7}$; increase in chronic disease prevalence and their complications will add drastically to this number. There is recent recognition of the potential for diabetes to reach epidemic proportions and the possible implications on vision impairment in India, especially as rural areas in India rapidly "urbanise." Although a population based study reported the prevalence of diabetic retinopathy in an urban population, ${ }^{8}$ data on diabetic retinopathy from India are primarily hospital based..$^{9-11}$ This paper reports on the prevalence of diabetic retinopathy among self reported diabetics in Palakkad district of Kerala in the southernmost part of India.

\section{PATIENTS AND METHODS}

The Palakkad Eye Disease Survey is a population based prevalence survey of visual impairment, blindness, and cataract surgical outcomes in a population aged 50 years and older of Palakkad district of the state of Kerala in southern India. We selected the survey population by cluster sampling. We constructed the sampling frame by grouping small villages together, and segmenting larger villages resulting in 1473 clusters with population ranging from 679 to 1800 . Twenty five clusters were randomly selected with equal probability, resulting in 22 rural and three urban clusters with an estimated 1991 census population of 320636 and approximately 54508 people $\geqslant 50$ years of age.

Fieldwork took place over an 8 week period starting in February 2001. Trained field workers working in four teams collected demographic details of subjects through a door to door enumeration of residents within the selected clusters. Residency was defined as having lived in the cluster for the past 6 months, including those temporarily absent. Those 50 years of age or older were invited to a site within the village for visual acuity measurement and ocular examination during a 2 day period immediately following the enumeration process. Two teams working independently at different sites performed clinical assessments; each team consisted of one ophthalmologist and two ophthalmic assistants.

Those physically unable to attend the examination site and those failing to come after repeated follow up contacts were offered the ocular examination at home. Those not willing to be examined either at home or at the examination site after a minimum of six follow up contacts were considered refusals. Eligible residents not present during the examination period were recorded as absent.

Presenting distance visual acuity and visual acuity with best correction after refraction were measured using illiterate $\mathrm{E}$ $\log$ MAR charts, distance visual acuity was measured at 4 metres and 1 metre. When necessary testing included the ability to count fingers, to detect hand movements, or to perceive light. "No light perception" was assigned to absent/ phthisical eyes. External eye and anterior segment examinations were performed using slit lamp biomicroscopy. Fundus examinations were performed using a direct ophthalmoscopy and indirect ophthalmoscopy using a 20D lens after dilatation of the pupils.

For reporting of vision status, we placed subjects in one of five vision categories: (1) NN: normal or near normal vision, $\geqslant 6 / 18$ in both eyes; (2) VI: unilateral or bilateral visual impairment, $<6 / 18$ to $\geqslant 6 / 60$ in the worse eye and $\geqslant 6 / 60$ in the better eye; ( 3 ) UL: unilateral blindness, $<6 / 60$ in the worse eye and $\geqslant 6 / 60$ in the better eye; (4) MB: moderate bilateral blindness, visual acuity $<6 / 60$ in the worse eye and $<6 / 60$ to $\geqslant 3 / 60$ in the better eye; (5) SB: severe bilateral blindness, visual acuity $<3 / 60$ in both eyes.

Assessment for diabetic retinopathy was independent of blood sugar levels in our study and based on a self reported 
Table 1 Demographic characteristics of 260 self reported diabetics of 5216 examined people; subjects aged 50 years and above

\begin{tabular}{llll}
\hline & No retinopathy & With retinopathy & Total \\
\cline { 2 - 4 } & No $(\%)^{*}$ & No $(\%)$ & No $(\%)$ \\
\hline Age (years) & $87(75.7)$ & $28(24.3)$ & $115(44.2)$ \\
$50-59$ & $71(75.5)$ & $23(24.5)$ & $94(36.2)$ \\
$\quad 60-69$ & $34(66.7)$ & $17(33.3)$ & $51(19.6)$ \\
$\quad 70$ & $99(72.3)$ & $38(37.7)$ & $137(52.7)$ \\
Sex & $93(75.6)$ & $30(24.4)$ & $123(47.3)$ \\
$\quad$ Male & $150(72.5)$ & $57(27.5)$ & $207(79.6)$ \\
$\quad$ Female & $42(79.2)$ & $11(20.8)$ & $53(20.4)$ \\
Literacy & $38(62.3)$ & $23(37.7)$ & $61(23.5)$ \\
$\quad$ Iliterate & $154(77.4)$ & $45(22.6)$ & $199(76.5)$ \\
$\quad$ Literate & $95(84.1)$ & $18(15.9)$ & $113(43.5)$ \\
Place of residence & $67(77.0)$ & $20(23.0)$ & $87(33.5)$ \\
$\quad$ Urban & $30(50.0)$ & $30(50.0)$ & $60(23.1)$ \\
$\quad$ Rural & $192(73.8)$ & $68(26.2)$ & $260(100.0)$ \\
Duration of diabetes (years) & & \\
$\quad<5$ & $5-10$ & & \\
$\quad>10$ & & & \\
Total & & &
\end{tabular}

history of diabetes. We elicited a history of current use of insulin to control diabetes. A modified classification of diabetic retinopathy based on the retinopathy levels used by Klein $e a^{12}$ was used in our study. Briefly, diabetic retinopathy was classified as non-proliferative diabetic retinopathy (NPDR), severe NPDR, and proliferative diabetic retinopathy (PDR). Non-proliferative diabetic retinopathy included levels $1-3$, severe NPDR included levels 4 and 5 , and proliferative diabetic retinopathy included levels 6 and 7 as described by Klein et al. The presence of clinically significant macular oedema (CSMO) was assessed using indirect and direct ophthalmoscopy. Presence of retinal photocoagulation scars was assessed using indirect ophthalmoscopy. Fundus photography was not performed because of the high costs involved and because the examinations were not hospital based and were performed at sites within villages. Subjects identified with severe NPDR, CSMO, or PDR were referred to the base hospital for more detailed examination including fluorescein angiograms and treatment.

Before the examination, a trained nurse measured the blood pressure of each study participant with a mercury column sphygmomanometer (Diamond Co Industrial Electronics and Allied Products, Electronics Cooperative estate, Pune, Maharashtra, India) using a standardised technique. ${ }^{13}$ Subjects were rested at least 5 minutes in a seated position before measuring the blood pressure. All blood pressure measurements were made on the left arm of each study subject, using a cuff of appropriate size at the level of the heart. The radial pulse was felt and the cuff level inflated $30 \mathrm{~mm} \mathrm{Hg}$ above the level at which the radial pulse disappeared and deflated slowly. The first and fifth Korotkoff sounds were recorded as systolic and diastolic blood pressure respectively. Systolic hypertension was defined either as a measured systolic blood pressure $=$ $140 \mathrm{~mm} \mathrm{Hg}$ and/or a diastolic blood pressure $90 \mathrm{~mm} \mathrm{Hg}$ or the current use of systemic antihypertensive medications.

Body mass index (BMI) measures were estimated from height and weight measurements of individual subjects; BMI was defined as weight $(\mathrm{kg}) /$ height $(\mathrm{m})^{2}$. Body weight was measured with the subject standing erect and motionless on the weighing scale without footwear and minimal outerwear, feet spaced $15 \mathrm{~cm}$ apart, and weight distributed equally. Height was measured with the subject in an erect position against a vertical surface, and with the head positioned level such that the external auditory meatus was level with the inferior margin of the bony orbit. Subjects were classified as lean if the BMI was $<20$ for males and $<19$ for females; as normal if the BMI was between 20-25 for males and between 19-24 for females; as overweight if the BMI was between 25-30 for males and between 24-29 for females, and obese if the BMI was $>30$ for males and $>29$ for females.

We used STATA version 7.0 software (College Station, TX, USA) for statistical analysis. Odds ratios (OR) and 95\% confidence intervals (95\% CI) are presented. Confidence intervals of the prevalence estimates have been calculated using a binomial approximation of normal distribution. $p$ Values less than 0.05 have been taken to indicate statistical significance.

Verbal informed consent was obtained from all participants at the examination site. The examination protocol was approved by the institutional review board of Aravind Medical Research Foundation, a unit of Aravind Eye Care System, Madurai, India, and was consistent with the principles laid down in the Helsinki declaration.

\section{RESULTS}

We enumerated 5666 people aged 50 years and above in 25 randomly selected clusters of Palakkad district. The response rate for participation in the study ranged from $88.3 \%$ to $96.6 \%$ across all clusters. We examined $5212(92.0 \%)$ of the 5666 subjects enumerated; 5071 (97.2\%) of the examined subjects were examined at the central examination site and an additional $145(2.8 \%)$ at their homes. The mean age of those examined was 62.0 years (range 50-101 years) and 2886 $(55.4 \%)$ were females.

Of the 5212 subjects examined, $260(5.0 \%)$ people gave a self reported history of diabetes. The mean age of self reported

Table 2 Classification of diabetic retinopathy among 260 people with self reported history of diabetes

\begin{tabular}{ll}
\hline & No $(\%)^{*}$ \\
\hline None & $192(73.8)$ \\
Mild NPDR & $40(15.4)$ \\
Moderate NPDR & $21(8.1)$ \\
Severe NPDR & $3(1.2)$ \\
CSMO & $20(7.7)$ \\
PDR & $4(1.5)$ \\
Total & $260(100.0)$ \\
\hline \multirow{2}{*}{ *Data presented as number of subjects (percentages). }
\end{tabular}


Table 3 Low vision and blindness among subjects with a self reported history of diabetes*

\begin{tabular}{|c|c|c|}
\hline & No diabetic retinopathy & Diabetic retinopathy \\
\hline & No (\%) $(95 \% \mathrm{Cl})$ & No $(\%)(95 \% \mathrm{Cl})$ \\
\hline \multicolumn{3}{|c|}{$(N N)$ Vision $\geqslant 6 / 18$ in both eyes } \\
\hline PVA† & $148(77.1)(70.7$ to 82.6$)$ & $42(62.7)(50.0$ to 73.9$)$ \\
\hline BCVA† & 171 (89.1) (83.6 to 93.0) & $47(70.2)(57.6$ to 80.4$)$ \\
\hline \multicolumn{3}{|c|}{ (VI) Vision $<6 / 18$ to $\geqslant 6 / 60$ in the worse eye and $\geqslant 6 / 60$ in the better eye } \\
\hline PVA & $28(14.6)(10.1$ to 20.6$)$ & 12 (17.9) (10.0 to 29.6$)$ \\
\hline BCVA & $8(4.2)(2.0$ to 8.3$)$ & 8 (11.9) (5.7 to 22.7$)$ \\
\hline \multicolumn{3}{|c|}{ (UL) Vision $<6 / 60$ in the worse eye and $\geqslant 6 / 60$ in the better eye } \\
\hline PVA & $10(5.2)(2.7$ to 9.6$)$ & 10 (14.9) (7.8 to 26.2$)$ \\
\hline BCVA & 11 (5.7) (3.0 to 10.3$)$ & $10(14.9)(7.8$ to 26.2$)$ \\
\hline \multicolumn{3}{|c|}{ (MB) Vision $<6 / 60$ in the worse eye and $<6 / 60$ to $\geqslant 3 / 60$ in the better eye } \\
\hline PVA & $4(2.1)(0.7$ to 5.6$)$ & $2(3.0)(0.5$ to 11.3$)$ \\
\hline BCVA & $1(0.5)(0.03$ to 3.3$)$ & $1(1.5)(0.08$ to 9.1$)$ \\
\hline \multicolumn{3}{|c|}{ (SB) Vision $<3 / 60$ in both eyes } \\
\hline PVA & $2(1.0)(0.7$ to 5.6$)$ & $1(1.5)(0.1$ to 9.1$)$ \\
\hline BCVA & $1(0.5)(0.03$ to 3.33$)$ & $1(1.5)(0.08$ to 9.1$)$ \\
\hline
\end{tabular}

diabetics was 61.7 (SD 8.0) years (range 50-86 years), and 137 $(52.7 \%)$ were males. Table 1 shows the characteristics of subjects in our study. A large proportion of the self reported diabetics were not on insulin therapy $(n=246,94.6 \%)$. A total of 169 people $(65 \%)$ of the self reported diabetics had diabetes for up to 10 years before our study.

The age-sex adjusted prevalence (based on projected population estimates for India for the year 2000-from the International Database of the US Census Bureau) of self reported diabetes in the 50 year age group was 5.1\% (95\% CI $3.9,6.3$, deff 4.33 ) and any diabetic retinopathy was $1.3 \%(95 \%$ CI: 0.8, 1.9 deff 2.69). The age-sex adjusted prevalence of diabetic retinopathy among the self reported diabetics was $26.8 \%$ (95\% CI: 19.2, 34.4 deff 1.99). The prevalence of diabetic retinopathy increased with increasing age although not at statistically significant levels even after adjusting for sex ( $\mathrm{p}$ value $=$ $0.264)$. The prevalence of diabetic retinopathy was not significantly associated with sex $(\mathrm{p}$ value $=0.540)$. Sixty four $(94.1 \%)$ of the 68 people diagnosed with diabetic retinopathy had NPDR of varying severity (Table 2 ) including 20 people $(29.4 \%)$ with CSMO. Twenty four (9.2\%) with diabetes required further treatment for retinopathy. Clinical features consistent with diabetic retinopathy were not detected in any of the people who did not give a history of diabetes.

Nine of the diabetics including three people who had diabetic retinopathy were presenting bilaterally blind at the $<6 / 60$ visual acuity level (Table 3 ). Blindness was attributed to diabetic retinopathy for two of these six eyes presenting bilaterally blind. After best correction with refraction, only four people remained bilaterally blind. Best corrected vision better than 6/18 was significantly higher among those without diabetic retinopathy compared with people with diabetic

Table 4 Causes for low vision and blindness among eyes with diabetic retinopathy

\begin{tabular}{ll}
\hline Causes & No (\%)* \\
\hline Refractive error & $7(38.9)$ \\
Cataract & $5(27.8)$ \\
Age related maculopathy & $2(11.1)$ \\
Clinically significant macular oedema & $2(11.1)$ \\
Posterior capsule opacification & $1(5.6)$ \\
Retinal detachment & $1(5.6)$ \\
Total & $18(100.0)$ \\
\hline \multirow{2}{*}{ *Data presented as number of subjects (percentages). }
\end{tabular}

retinopathy $(\mathrm{p}=0.03)$. Table 4 shows the causes for low vision and blindness among eyes with diabetic retinopathy. Cataract and refractive errors remained the major causes for low vision and blindness in these subjects.

Thirty two (12.3\%) people with diabetes were obese. On univariate analysis, body mass index was not associated with diabetic retinopathy ( $p$ value $=0.317)$. A total of $184(72.4 \%)$ people with diabetes had associated hypertension including $47(74.6 \%)$ people with diabetic retinopathy. There was no significant association between diabetic retinopathy and hypertension in univariate analysis ( $\mathrm{p}$ value $=0.658$ )

Multivariate analysis using a multiple logistic regression model was used to explore possible risk factors for diabetic

Table 5 Relative odds ( $95 \%$ confidence intervals) for risk factors for diabetic retinopathy

\begin{tabular}{|c|c|c|}
\hline & Relative odds & $\begin{array}{l}\text { Adjusted relative } \\
\text { odds }\end{array}$ \\
\hline \multicolumn{3}{|l|}{ Age (years) } \\
\hline $50-59$ & 1 & 1 \\
\hline $60-69$ & $1.00(0.53$ to 1.90$)$ & $1.20(0.57$ to 2.52$)$ \\
\hline$>70$ & $1.55(0.76$ to 3.20$)$ & $1.38(0.59$ to 3.20$)$ \\
\hline \multicolumn{3}{|l|}{ Sex } \\
\hline Male & 1 & 1 \\
\hline Female & $0.84(0.48$ to 1.47$)$ & $1.02(0.51$ to 2.05$)$ \\
\hline \multicolumn{3}{|l|}{ Literacy } \\
\hline Illiterate & 1 & 1 \\
\hline Literate & $0.69(0.33$ to 1.43$)$ & $0.81(0.34$ to 1.94$)$ \\
\hline \multicolumn{3}{|c|}{ Place of residence } \\
\hline Urban & 1 & 1 \\
\hline Rural & $0.48(0.26$ to 0.89$)$ & $0.68(0.31$ to 1.50$)$ \\
\hline \multicolumn{3}{|c|}{ Duration of diabetes (years) } \\
\hline$<5$ & 1 & 1 \\
\hline $5-10$ & $1.58(0.76$ to 3.20$)$ & $1.24(0.58$ to 2.66$)$ \\
\hline$>10$ & $5.28(2.58 \text { to } 10.78)^{*}$ & $3.01(1.32$ to 6.86$)$ * \\
\hline \multicolumn{3}{|c|}{ Current use of insulin } \\
\hline No & 1 & 1 \\
\hline Yes & $\begin{array}{l}12.16(3.28 \text { to } \\
45.08)^{*}\end{array}$ & $\begin{array}{l}10.56(1.15 \text { to } \\
97.47)^{*}\end{array}$ \\
\hline \multicolumn{3}{|c|}{70.001} \\
\hline Normal & 1 & 1 \\
\hline Lean & $0.72(0.22$ to 2.35$)$ & $0.72(0.19$ to 2.70$)$ \\
\hline Overweight & $0.92(0.48$ to 1.79$)$ & $0.87(0.42$ to 1.81$)$ \\
\hline Obese & $0.36(0.12$ to 1.11$)$ & $0.46(0.14$ to 1.49$)$ \\
\hline \multicolumn{3}{|l|}{ Hypertension } \\
\hline No & 1 & 1 \\
\hline Yes & $1.16(0.61$ to 2.21$)$ & $1.01(0.47$ to 2.16$)$ \\
\hline
\end{tabular}


retinopathy. Duration of diabetes longer than 10 years and current use of insulin were significantly associated with diabetic retinopathy (Table 5).

\section{DISCUSSION}

Diabetic retinopathy is one of the few ophthalmic diseases that have a defined preventive measure to delay progression of the disease and consequent visual loss. The estimated high prevalence of diabetes by 2025 in India $^{6}$ is a matter of concern considering the potential for vision loss associated with diabetic retinopathy.

Our study is limited in that we relied on a self reported history of diabetes and did not perform fasting blood glucose measurements or glycosylated haemoglobin measurements. It is probable that the $5.1 \%$ of diabetes estimated in this population is an underestimate; we may have failed to detect previously undiagnosed diabetics owing to the lack of blood glucose measurements in our study. The lack of fundus photographs is also a limitation of our study; it is possible that we may have missed some early diabetic retinopathy and underestimated the prevalence of diabetic retinopathy. We were unable to perform a stereoscopic evaluation of the macula as we used a portable hand held slit lamp for biomicroscopy; this may have led to a possible underestimation of CSMO. A strength of our study is the door to door enumeration and high participation rates among those enumerated coupled with the random nature of the sample, supporting the generalisability of results from this study to the population of Palakkad district.

The age-sex adjusted prevalence of diabetic retinopathy among those aware of their diabetic status in our study is nearly similar to the $22.4 \%$ diabetic retinopathy among self reported diabetics in the urban population of Andhra Pradesh in India, ${ }^{8}$ and to the $34.1 \%$ prevalence of diabetic retinopathy (the confidence intervals overlap) reported from a series of diabetics attending a diabetes centre in urban southern India. ${ }^{9}$ The prevalence reported by us is much lower than the $52 \%$ reported in the Melton study, ${ }^{14}$ but is comparable to most other large population based studies. ${ }^{15-19}$

Previous studies have established the vision threatening potential of diabetic retinopathy ${ }^{3}$; we found 18 eyes of nine people to be presenting visual acuity blind at the $<6 / 60$ level, however, only two of these eyes were blind due to diabetic retinopathy. Cataract remained a major cause for blindness in these eyes. While the surgical removal of cataract may restore vision to patients blind from cataract, there is potential for progression of diabetic retinopathy after cataract surgery suggesting that these patients remain at an increased risk for blindness even if their cataracts are removed ${ }^{20-25}$ Although we found best corrected vision better than $6 / 18$ to be significantly higher among those without diabetic retinopathy, the small number of subjects in our study does not allow us adequate statistical power to make this inference.

We did not find any significant association between diabetic retinopathy and age, sex, BMI, and hypertension. However, we did not have sufficient cases of diabetic retinopathy to give us adequate statistical power to identify weak associations. Consistent with other studies, we found diabetic retinopathy to be associated with duration of diabetes and use of insulin to control diabetes.

The relatively low prevalence of diabetic retinopathy (1.3\%) and blindness due to diabetic retinopathy in this population aged 50 years and older may suggest that diabetic retinopathy requires less priority and attention than the other major visual impairing diseases in India-like cataracts and refractive errors-that account for nearly $90 \%$ of the current burden of blindness in India. However, it has to be realised that the projected 57 million diabetics by 2025 in India may drastically alter the existing pattern of blindness in India. Improving healthcare facilities in India will probably translate into a large number of diabetics living longer, and thus more diabetics at risk for developing retinopathy. If retinopathy is not detected and treated early, the potential for vision loss is high, this will add to the burden of needless visual impairment and blindness in India. This will require improved networking between ophthalmologists and internists in India such that all diabetic patients receive a dilated fundus examination at regular intervals.

Current treatments for diabetic retinopathy including lasers are vision preserving at best, compared with the vision restoring potential for cataract surgeries or spectacles. The current costs of intervention for diabetic retinopathy is higher than the costs for treating either cataracts or refractive errors, and higher than the costs involved for preventing or delaying onset of diabetic retinopathy. If $10 \%$ (as suggested by our data) of the projected 57 million diabetics by 2025 develop severe retinopathy or CSMO, it will mean that 5.7 million people will require either laser or surgical intervention. This will be in addition to treatment required for more prevalent visual impairing diseases including cataracts and refractive errors. Tackling this burden will require a larger number of trained personnel besides adequate infrastructure support. Training to treat vitreoretinal diseases including diabetic retinopathy is currently not part of all ophthalmology residency programmes, such training is often offered as post-residency fellowships in India. Few institutes in India offer post-residency fellowships; the number of ophthalmologists opting for postresidency fellowship training is also not sizeable. The challenge this will pose to the ophthalmic healthcare system in India is underscored when we consider that a cataract focused national policy to prevent blindness in India has been able to increase annual cataract surgeries only to 3.5 millions/ year in 2000 (data from Ministry of Health and Family Welfare, Government of India) after at least a decade of sustained effort, despite training to perform cataract surgeries being part of every ophthalmic residency programme.

There are only two ways to prevent blindness from retinopathy in people with diabetes (1) maintaining a strict glycaemic control, and (2) regular ophthalmic examinations of those identified as diabetic to detect early retinopathy. Broadening the focus of existing community screening programmes to include screening for diabetic retinopathy should be considered for early detection of retinopathy especially among the underserved populations. Formulating national policy guidelines aiming at preventing or delaying the onset of diabetic retinopathy will ensure that diabetic retinopathy does not become a major cause for needless visual impairment or blindness in the future.

Further studies are required to determine the changing magnitude of diabetic retinopathy and diabetes, as well as to understand the risk factors for diabetic retinopathy and visual loss in this population. The current low prevalence of diabetic retinopathy in India might necessitate such studies being part of other large population based studies or hospital based studies

\section{ACKNOWLEDGEMENT}

The study was supported by the Aravind Medical Research Foundation, Aravind Eye Care System, Madurai, India.

\section{Authors' affiliations \\ V Narendran, R K John, A Raghuram, R D Ravindran, P K \\ Nirmalan, R D Thulasiraj, Aravind Medical Research Foundation, Aravind Eye Care System, Tamil Nadu, India \\ Financial interest: None. \\ REFERENCES \\ 1 Palumbo RJ, Elveback LR, Chu CP, et al. Diabetes mellitus: incidence, prevalence, survivorship and causes of death in Rochester, Minnesota, 1945-1970. Diabetes 1975;25:566-73.}


2 Butler WJ, Ostrander LD, Carman WJ, et al. Diabetes mellitus in Tecumseh, Michigan: prevalence, incidence and associated conditions. Am J Epidemiol 1982;116:971-80.

3 Aiello LM, Rand LI, Brones JC, et al. Diabetic retinopathy in Joslin Clinic patients with adult-onset diabetes. Ophthalmology 1981;88:619-23.

4 West KM, Erdriech L, Stober JA. A detailed study of risk factors for retinopathy and nephropathy in diabetes. Diabetes 1980;29:501-8.

5 World Health Organization. Global initiative for the elimination of avoidable blindness. An informal consultation. WHO/PBL/97.61. Geneva: WHO, 1997.

6 King $\mathbf{H}$, Aubert RE, Herman WH. Global burden of diabetes, 1995-2025: prevalence, numerical estimates, and projections. Diabetes Care 1998;21:1414-31.

7 Thylefors B, Negrel AD, Pararajasegaram R, et al. Global data on blindness. Bull World Health Organ 1995:73:1 15-21.

8 Dandona L, Dandona R, Naduvilath TJ, et al. Population based assessment of diabetic retinopathy in an urban population in southern India. Br J Ophthalmol 1999;83:937-40.

9 Rema M, Ponnaiya M, Mohan V. Prevalence of retinopathy in non insulin dependent diabetes mellitus at a diabetes center in Southern India. Diabetes Res Clin Pract 1996;34:29-36.

10 Ramachandran A, Snehalatha C, Vijay V, et al. Diabetic retinopathy at the time of diagnosis of NIDDM in south Indian subjects. Diabetes Res Clin Pract 1996:3:111-4.

11 Rema M, Deepa R, Mohan V. Prevalence of retinopathy at diagnosis among type 2 diabetic patients attending a diabetic center in south India. Br J Ophthalmol 2000;84: 1058-60.

12 Klein R, Klein BEK, Magli YL, et al. An alternative method of grading diabetic retinopathy. Ophthalmology 1986;93:1 183-7.

13 Perloff D. Human blood pressure determination by sphygmomanometry. Circulation 1993;88:2460-70.

14 Sparrow JM, McLeod BK, Smith TD, et al. The prevalence of diabetic retinopathy and maculopathy and their risk factors in the non-insulin-treated diabetic patients of an English town. Eye 1993;7:158-63.
15 Mitchell P, Smith W, Wang JJ, et al. Prevalence of diabetic retinopathy in an older community: the Blue Mountains Eye Study. Ophthalmology 1998; 105:406-11.

16 Stolk RP, Vingerling JR, de Jong PT, et al. Retinopathy, glucose and insulin in an elderly population: the Rotterdam study. Diabetes 1995;44:11-15.

17 Klein R, Klein BEK, Moss SE, et al. The Beaver Dam Study: Retinopathy in adults with newly discovered and previously diagnosed diabetes mellitus. Ophthalmology 1992;99:58-62.

18 McCarty CA, Lloyd-Smith CW, Lee SE, et al. Use of eye care services by people with diabetes: the Melbourne Visual Impairment Project. $\mathrm{Br} J$ Ophthalmol 1998;82:410-4

19 McKay R, McCarty CA, Taylor HR. Diabetic retinopathy in Victoria, Australia: the Visual Impairment Project. Br J Ophthalmol 2000;84:865-70

20 Pollack A, Dotan S, Oliver M. Progression of diabetic retinopathy after cataract extraction. Br J Ophthalmol 1991;75:547-51.

21 Pollack A, Leiba H, Bukelman A, et al. The course of diabetic retinopathy following cataract surgery in eyes previously treated by laser photocoagulation. Br J Ophthalmol 1992;76:228-31.

22 Cunliffe IA, Flanagan DW, George NDL, et al. Extracapsular cataract surgery with lens implantation in diabetics with and without proliferative retinopathy. Br J Ophthalmol 1991;75:9-12.

23 Ruiz RS, Saatci OA. Posterior chamber intraocular lens implantation in eyes with inactive and active proliferative diabetic retinopathy. Am J Ophthalmol 1991;111:158-62.

24 Hykin PG, Gregson RM, Stevens ID, et al. Extracapsular cataract extraction in proliferative diabetic retinopathy. Ophthalmology 1993; 100:394-9.

25 Benson WE, Brown GC, Tasman W, et al. Extracapsular cataract extraction with placement of a posterior chamber lens in patients with diabetic retinopathy. Ophthalmology. 1993;100:730-8.

\section{New mutations cause congenital cataracts}

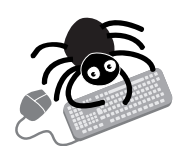

Please visit the British Journal of

Ophthalmology website [www. bjophthalmol.com for link to this full article.
$\mathrm{N}$

ew mutations in $\gamma$-crystallin $(C R Y G)$ genes cause congenital cataracts in Indian families. The discovery, which mirrors the influence of cryg genes in mice, may throw light on how cataracts form.

A study of seven families with an index case of cataract in both eyes in a child identified three point mutations in the CRYG gene cluster. These segregated with cataracts in close relatives of the index case in an autosomal dominant pattern of inheritance. Two mutations were missense mutations in the CRYGC and $C R Y G D$ genes. They were associated with lamellar cataracts. One mutation was a nonsense mutation in $C R Y G D$ associated with central nuclear cataracts. In four families mutations were not evident in $C R Y G$ genes and are yet to be identified.

The index cases were selected from children aged under 15 years presenting to a paediatric eye clinic in southern India with cataracts in both eyes. Mutations were identified by amplification and sequence

] analysis of the $\gamma$-crystallin gene cluster in genomic DNA samples taken from the index cases and their close relatives.

Cataracts in both eyes are more common in Indian children than in other ethnic groups. The $\gamma$-crystallin genes are crucial for the development of the lens of the eye as they specify one of its main protein components. Their sequences are highly conserved in a mammals, making them obvious candidates for study.

A Journal of Medical Genetics 2002;39:352-358. 